SPATIAL COGNITION AND COMPUTATION, 6(4), 345-368

Copyright @ $\odot$ 2006, Lawrence Erlbaum Associates, Inc.

\title{
Spatial Memory Organized by Environmental Geometry
}

\author{
Thomas Schmidt \\ University of Göttingen \\ Eun Young Lee \\ University of Missouri-Columbia
}

We investigated the simultaneous effects of different reference systems on spatial memory. Participants studied a configuration of objects surrounding them. During retrieval, they imagined themselves in the center of the object configuration facing a particular object, and then indicated the directions of other objects relative to this imagined heading. Besides strong effects of egocentric retrieval direction, retrieval was enhanced for objects and headings aligned with an object-centered reference system (triangular object configuration within a neutrally-shaped room), or with a sufficiently salient environmental reference system (triangular room surrounding a neutrally-shaped object configuration). Moreover, remembered object positions were spatially distorted by the object-centered reference system. Results suggest that object positions are accessed by imagining oneself within a topographical representation of objects which is preorganized in terms of both environmental and object-centered reference systems.

Keywords: Spatial cognition, spatial memory, spatial reference systems, spatial memory distortions.

\section{Introduction}

In complex environments containing many spatial cues, it is clearly uneconomical to memorize each possible path from one object to another,

Correspondence concerning this article should be addressed to Thomas Schmidt, University of Giessen, Department of Cognitive Psychology, Otto-Behaghel-Str. 10F, D35394 Giessen, Germany; email: thomas.schmidt@psychol.uni-giessen.de. 
346 SCHMIDT, LEE

because there would be a combinatorial explosion of paths to learn. Instead of explicitly representing all these paths, it would be advantageous to use a topographical representation storing only the spatial layout of objects, and having a means of reconstructing arbitrary paths from this implicit representation. Importantly, such mental maps (Tolman, 1948) might contain a representation of one's own body position as well, so as to allow for the positions of objects to be encoded either relative to other objects, relative to an extraneous set of environmental landmarks, or relative to the own body, making it possible to flexibly change from one such spatial reference system to another (Levinson, 1996).

In many areas of cognitive psychology and neuroscience, reference systems are classified as either egocentric or allocentric (e.g., Milner \& Goodale, 1995). Egocentric reference systems specify spatial information in relation to the observer's body or viewing perspective, which is crucial for performing the coordinate transformations necessary for spatially directed actions such as grasping, pointing, or walking towards an object (Flanders, Helms, Tillery, \& Soechting, 1992). Allocentric reference systems, on the other hand, use some external frame of reference that is independent of the observer's position or orientation. In this paper, we distinguish between two types of allocentric reference systems, following Carlson-Radvansky and Irwin (1993). First, environment-centered reference systems are anchored to features of the target object's surroundings (like salient landmarks in an open landscape, or room geometry) independently of the position or orientation of the object itself (Pani \& Dupree, 1994; Shelton \& McNamara, 2001). In contrast, object-centered reference systems (also called intrinsic; Levinson, 1996; Schmidt, 2004) are locked to geometric features of the target object, e.g., its axes of elongation, symmetry, or movement direction. Shelton and McNamara (2001) suggest that reference systems are crucial for learning and remembering the spatial structure of a surrounding environment: If a salient reference system is available, privileged directions are created in spatial memory that can later be accessed more quickly or more reliably.

Inferring reference systems from response speed and accuracy. Clear evidence for the importance of egocentric reference systems in spatial memory comes from a series of experiments by Hintzman, O'Dell, and Arndt (1981). Participants learned a configuration of eight objects circularly arranged around them. During the retrieval phase, they had to imagine themselves in the middle of the configuration facing a particular object, and had to point towards the location of other objects relative to that imagined orientation. Objects imagined in front of the participants were retrieved quickly but objects on the sides were retrieved increasingly slower as their angular distance from the imagined straight-ahead increased. However, objects imagined directly behind the participants were identified almost as quickly as those in front. Similarly, 
Franklin and Tversky (1990; see also Franklin \& Tversky, 1992; Franklin, Tversky, \& Coon, 1992; Mou, Zhang, \& McNamara, 2004) could show that reaction times are fastest for above-below judgments, followed by front-back and finally by left-right judgments of spatial relations learned from narrative texts.

Shelton and McNamara (Diwadkar \& McNamara, 1997; Roskos-Ewoldsen, McNamara, Shelton, \& Carr, 1998) showed that the egocentric viewpoints from which spatial configurations are learned greatly affect later retrieval. Participants learned two orthogonal views of an irregular assembly of seven objects from outside positions within a large square room. In a later test phase, they had to retrieve the direction of objects from imaginary viewpoints defined by other objects (for example, "Imagine you are at the bottle and facing the shoe -- point to the ball."). Pointing judgments were faster and more accurate when the participants' imagined heading was parallel to one of the two views actually studied, compared to unfamiliar views. Later studies showed these viewpointdependency effects to be surprisingly robust, because as many as three alternative outside views of an object configuration did not suffice to abolish them in favor of an orientation-independent representation (Shelton \& McNamara, 2001).

Whereas all these results stress the importance of egocentric reference systems in spatial memory organization, Werner, Saade, and Lüer (1998) could show that both allocentric and egocentric reference systems are used in representing room-sized environments (see Hermer \& Spelke, 1994, 1996, for a developmental perspective). Participants learned a configuration of eight objects arranged circularly around them in a nearly square room, one object being placed in front of each wall or corner. In the test phase, participants were told to imagine themselves facing one of the walls or corners; when a sound was presented from one of the eight directions, participants had to name the corresponding object while pressing a response key at the same time. Participants could name objects faster and more reliably the closer the retrieved direction was to the current imagined orientation, with an additional advantage for positions imagined directly behind, indicating the influence of an egocentric reference system as demonstrated earlier by Hintzman et al., (1981). In addition, however, participants were faster and made fewer errors when imagining themselves facing a wall than facing a corner, suggesting that an environmentbased allocentric reference system was superimposed on the egocentric one. Other studies also suggest that salient geometric features are spontaneously used in large-scale natural environments (McNamara, Rump \& Werner, 2003; Werner \& Schmidt, 1999; Werner \& Schmidt, 2006) and in learning from maps (Levine, Jankovic, \& Palij, 1982; Sholl, 1987).

In a series of experiments, Shelton and McNamara (2001; see also Burgess, Spiers, \& Paleologou, 2004; Wang \& Simons, 1999) could demonstrate strong 


\section{SCHMIDT, LEE}

interactions between environmental and viewpoint-based reference systems. When object configurations had to be retrieved from imaginary viewpoints, retrieval was best when the imagined viewing perspective matched the one actually encountered during learning. However, whenever participants learned the configuration from more than one viewpoint, only those views aligned with one or more environmental reference systems (rectangular carpet or room) were favored; alignment was not important when only one perspective was learned. Furthermore, Mou and McNamara (2002) showed that retrieval was enhanced for imagined headings parallel to the symmetry axis of an object configuration even if those views were never encountered during learning (Mou, McNamara, Valiquette, \& Rump, 2004; Wang \& Brockmole, 2003). These results indicate that participants are able to switch between actual and imagined headings as well as different types of reference system.

Inferring reference systems from spatial memory distortions. Response times and accuracy rates are indirect means of identifying the reference systems of an underlying spatial memory representation. More direct information about these representations can be obtained by asking participants to reproduce the remembered positions of spatial objects, e.g., following a brief visual presentation (Huttenlocher, Hedges, \& Duncan, 1991; Laeng, Peters, \& McCabe, 1998; Nelson \& Chaiklin, 1980). For instance, (Diedrichsen, Werner, Schmidt, \& Trommershäuser, 2004; Schmidt, Werner, \& Diedrichsen, 2003; Werner \& Diedrichsen 2002) showed observers a small target dot in the presence of two horizontally aligned circles (visual landmarks), one to the left and one to the right of display center, in an otherwise empty display. After a variable retention interval where the display was visually masked, the landmarks reappeared, and the observers had to use a mouse cursor to reproduce the exact location of the target dot relative to the landmarks. It was found that the landmarks induced a complex and symmetrical pattern of spatial biases, called a distortional field: Targets near the landmarks were reproduced too far away from them as if repulsed in a radial fashion, and there was an additional bias away from the midpoint between the landmarks as if it formed a virtual landmark (Schmidt et al., 2003).

Trommershäuser and Schmidt (in preparation) propose that spatial memory distortions arise from attentional processes in topographical spatial memory maps where reference points and lines represent those regions of the map tending to receive the strongest deployment of spatial attention. This notion helps explaining the genesis of spatial distortions. Suzuki and Cavanagh (1997) have shown that a flanking dot presented next to a vertical line can make the line appear to be shifted away from the flanker, but only if the flanker is visually attended. The authors propose that spatial distortions away from attended distractors might occur in a topographic array of visual cells by several types of interactions between neighboring units, such as the narrowing of spatial tuning 
curves (Moran \& Desimone, 1985; Spitzer, Desimone, \& Moran, 1988), the shifting of receptive fields towards the focus of attention (Connor, Preddie, Gallant, \& Van Essen, 1997), and the inhibition of cells outside the attentional focus by those inside the focus.

Distortional fields have been employed to infer one or more of the reference systems underlying a spatial memory representation. This can be done by examining distortional fields for symmetry about different points or axes (Schmidt et al., 2003; Schmidt, 2004). For instance, when target dots are reproduced relative to a configuration of landmarks forming an equilateral triangle, symmetry properties of distortional fields depend on which of the three symmetry axes of the triangle coincides with a natural extrinsic reference system, such as the allocentric vertical axis (Schmidt, 2004). The strong symmetry of the distortional field about the allocentric vertical indicates that this axis is used as a spatial reference.

Overview of the experiments. Our goal here was to study interactions of egocentric, environment-centered, and object-centered reference systems, taking into account the possibility that reference systems might reveal themselves in terms of spatial memory distortions in addition to traditional measures of retrieval speed and accuracy. We adopted the paradigm of Hintzman et al., (1981) and Werner et al., (1998) by having observers learn an object configuration from its center such that all objects were spaced at equal angles. During retrieval, the observers' task was to mentally align themselves with a given target direction and then indicate the position of other objects relative to that direction by pointing a joystick as quickly and accurately as possible. In Experiment 1, we studied object configurations in a spatially neutral environment, a circular room. Allocentric reference systems were introduced by employing either a triangular carpet (an environmental reference system) or a triangular object configuration arranged along the edges of the triangular carpet (environmental plus object-centered reference system). In Experiment 2, we focused on a more salient environmental reference system by using a triangular room surrounding a circular configuration of objects.

We focussed on several independent aspects of performance. Foremost, we expected an egocentric effect such that retrieval times should vary with respect to the position of the retrieved object relative to the imagined heading, with increasingly slower access for object positions further away from the imagined heading (Hintzman et al., 1981). In addition, several predictions were made regarding allocentric effects induced by object-centered and/or environmental reference systems. First, we expected faster retrieval when the imagined heading was aligned with a salient environmental axis (e.g., the top or base of the triangle; Werner et al., 1998). Second, we expected objects located on salient environmental axes to be retrieved faster than objects on other axes, irrespective of imagined heading. Third, we expected that if any distortion of object 
positions in spatial memory was induced by the triangular reference system, this distortion pattern should be symmetrical with respect to the major axis of the environmental reference system, thus providing independent evidence that this axis was used as a spatial reference.

\section{Experiment 1}

The experiment consisted of two phases. In a study phase, participants were seated on a swivel chair in the center of a round room furnished with a triangular carpet. They learned the locations of eight objects arranged around them either in a circle or along the edges of the carpet (Figure 1a). In a test

a) Experiment 1

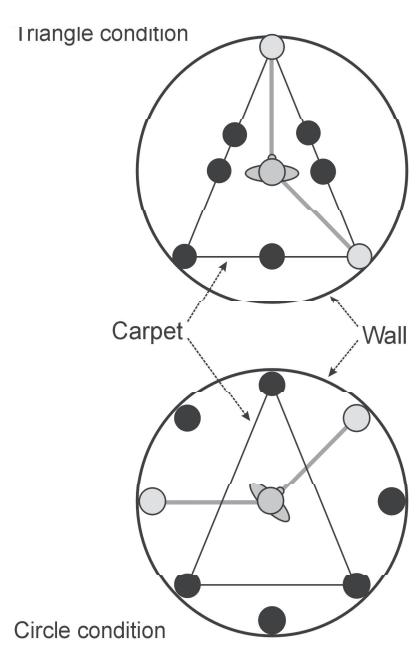

b) Experiment 2

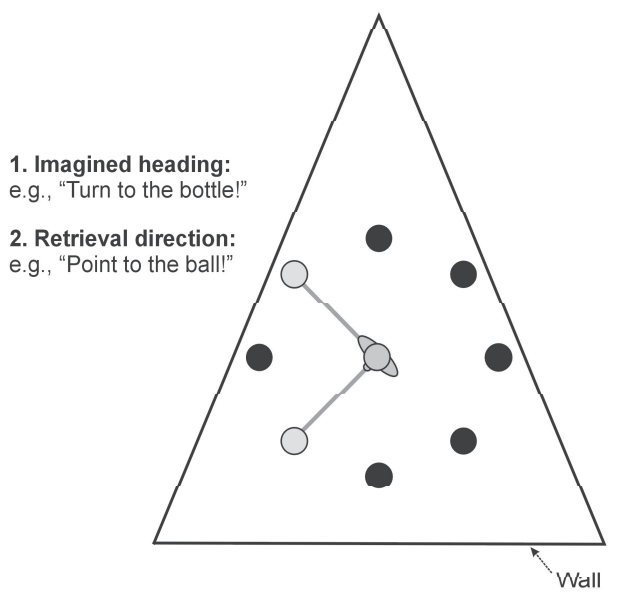

Figure 1. Experimental setup in Experiment 1. Learning took place in a circular room furnished with a triangular carpet. Objects were placed either along the edges of the carpet (triangular condition, upper panel) or along the walls of the room (circular condition, lower panel); in both cases, adjacent objects had constant angular spacings with respect to the participant who was placed in the center of the room. During each trial in the retrieval phase, participants imagined themselves looking at a particular object, retrieving the locations of other objects relative to this imagined heading. $b$ : Experimental setup in Experiment 2. Learning took place with circular object configurations in a triangular room. No carpet was employed. 
phase, which took part in another room, participants had to imagine themselves oriented towards one of the objects and to retrieve the other objects (targets), one by one, relative to this imagined heading, by moving a joystick in the appropriate direction. Apart from the commonly observed egocentric effects of object direction relative to the imagined heading, we hypothesized that under neutral room geometry only the environmental reference frame anchored to the carpet and the object-centered reference frame anchored to the object configuration could take effect on spatial memory representations. We further expected these allocentric effects to be stronger if objects were assembled along the edges of the carpet (environmental plus object-centered reference system) than when the configuration was circular (environmental reference system only). Thus, the design was able to reveal egocentric effects, environmental effects, and a possible enhancement of environmental effects by object-centered effects.

\section{Methods}

Participants. 32 students from the University of Göttingen (12 men, 20 women, age 18 to 36 years) participated for course credit or for a payment of $€$ 15. All of them gave informed consent.

Apparatus and stimuli. For the study phase, a gray-colored carpet shaped as an isosceles triangle with edge lengths of 230 and $176 \mathrm{~cm}$ was placed in a round chamber, $245 \mathrm{~cm}$ in diameter, so that the corners of the triangle were touching the walls. The round chamber was built out of thick drapery that covered the walls, floor, and ceiling, and was set up in a darkened larger room. The chamber was accessed through a flap in the wall that was concealed with fabric once the participant had entered. The participant's seat was in the center of the room. Eight objects ("ball", "shoe", "can", "phone", "hat", "bag", "bottle", and "pot") were arranged around the seat according to two different conditions: In the circle condition, objects were arranged along the walls of the room; in the triangle condition, they were arranged along the edges of the triangular carpet (Figure 1a). In both conditions, objects were placed at $45^{\circ}$ intervals, so that corresponding objects from the two conditions always required identical angular responses. All objects were easy to discriminate from each other.

The test phase took place in a customary, rectangular laboratory room. The experiment was conducted on a Macintosh 7600/132 computer with a 17" monitor. Acoustic stimuli were spoken German object names that had been electronically edited to have a duration of $250 \mathrm{~ms}$. Participants used a Gravis200 joystick mounted on a table before them. The joystick was chosen because it offered the same mechanical resistance in all angular directions.

Procedure. Participants were randomly assigned to the triangle or circle conditions. First, they were led blindfolded to the center of the round room and seated on the swivel chair; they turned themselves on the chair until they felt 
disoriented. They then studied the locations of the eight objects, rotating themselves upon instruction from one heading to an adjacent one until the configuration had been studied eight times, once from each possible orientation, for one minute each. After that, they were allowed to study the object configuration freely. Half of the participants changed viewpoints in clockwise, the other half in counterclockwise order, with the starting direction counterbalanced across participants. Participants were then blindfolded once more and led out of the room. The study phase lasted about 10-12 min.

The test phase took part in a different room immediately after the learning phase. Before the start of the main session, participants had to enumerate all studied objects in the correct sequence. They were then made familiar with the use of the joystick by having to point as quickly and accurately as possible to eight watch-hand directions ("twelve o'clock", "half past one", etc.) in a practice block. Visual feedback about response time and angular deviation was given after each trial.

In the test phase proper, participants were instructed to imagine themselves sitting in the center of the round room, looking at a particular object and retrieving the spatial locations of the other objects. Participants initiated each trial by pressing the joystick trigger. Each trial started with an acoustic instruction "Please turn to..." (in German) followed by the name of the object. Participants had to mentally align themselves with this object and press a joystick key when ready. The name of the target object was then presented for $250 \mathrm{~ms}$, and the participant had to indicate its direction relative to the imagined heading by moving the joystick in the recalled direction as accurately and quickly as possible, with the joystick's straight-ahead direction defined as the direction of imagined heading. A response was registered as soon as the joystick was moved to its limit stop, and response time and pointing direction were recorded. After each trial, participants received visual feedback about their response time and angular deviation from the correct target. After all eight possible targets (including the straight-ahead object) were retrieved once in random order, a new mental orientation was assumed.

Participants performed three blocks of 64 trials each. In each block, they mentally aligned themselves with all eight possible orientations once, in random order, and were asked to reproduce the locations of all eight possible target objects on the basis of this heading, so that every combination of imagined heading and retrieved target occurred once in each block. The test phase took about half an hour. After the main experiment, some of the participants were asked to draw the object configuration, and they were all informed about the purpose of the study.

Statistical methods and coding conventions. Trials with an angular error exceeding a predetermined criterion of $\pm 90^{\circ}$ were excluded from analysis (6.6 $\%$ ), as were trials with response times more extreme than \pm 3 standard deviations 
(1.5\%) from the mean (calculated separately for each participant). ${ }^{1}$ Response times (from target onset to completion of the joystick response) as well as signed angular pointing error were submitted to two-way analyses of variance separately for the circle and triangle conditions (ANOVAs; mixed model, Greenhouse-Geisser adjusted $p$ values, Bonferroni-corrected $\alpha / 2=.025$ ) having within-subject factors of retrieval direction (relative to the imagined heading, egocentric effect) and alignment (of imagined heading and triangular carpet, allocentric effect). Retrieval direction of $0^{\circ}$ indicated that the target direction coincided with the imagined heading; imagined heading of $0^{\circ}$ indicated that the heading was towards the top of the triangle. Imagined heading was coded as aligned with the carpet for headings of $0^{\circ}$ or $180^{\circ}$, and misaligned otherwise. Angles were measured clockwise.

\section{Results}

Response times. Effects of an egocentric reference system were analyzed by looking at the retrieval times of objects at various positions with respect to the imagined heading. In the triangle condition (Figure $2 \mathrm{a}$ ), there was a strong main effect of retrieval direction, $F(7,105)=12.22, p<.001$, indicating that retrieval was fastest for objects imagined straight ahead and slower for objects located increasingly far apart from the imagined heading. Objects imagined straight ahead were retrieved faster than objects at any other position (planned contrast at $\alpha / 2=.025$, pooled error term), $F(1,105)=37.57, p<.001)$, and objects imagined straight behind tended to be retrieved faster than the two neighboring objects at $135^{\circ}$ and $225^{\circ}, F(1,105)=3.96, p<.05$ (not significant after $\alpha$ correction). In the circle condition (Figure $2 b$ ), the same effect of retrieval direction was found, $F(7,105)=18.09, p<.001$. Again, objects imagined straight ahead were retrieved faster than all other objects, $F(1,105)=53.12, p<$ .001 , whereas the advantage of objects imagined straight ahead over the two neighboring positions was not significant, $F(1,105)=2.71, p>.1$.

Two types of allocentric effects on response times were analyzed. First, we looked at retrieval times when the imagined heading was aligned or misaligned with the triangular reference system. In the triangle condition, retrieval was indeed faster for aligned than for misaligned headings, $F(1,15)=28.54, p<$ .001 , and this effect interacted with retrieval direction, $F(7,105)=4.30, p=$ .007 , mainly reflecting an advantage for objects imagined straight behind which

\footnotetext{
${ }^{1}$ Because objects were spaced $45^{\circ}$ apart, a cut-off criterion of $22.5^{\circ}$ would have been appropriate to assign responses unambiguously to single objects. Adoption of such a strict criterion led to unreasonably large proportions of data dropping out of analysis. However, the pattern of means largely remained unchanged when a strict criterion was employed.
} 


\section{Experiment 1}
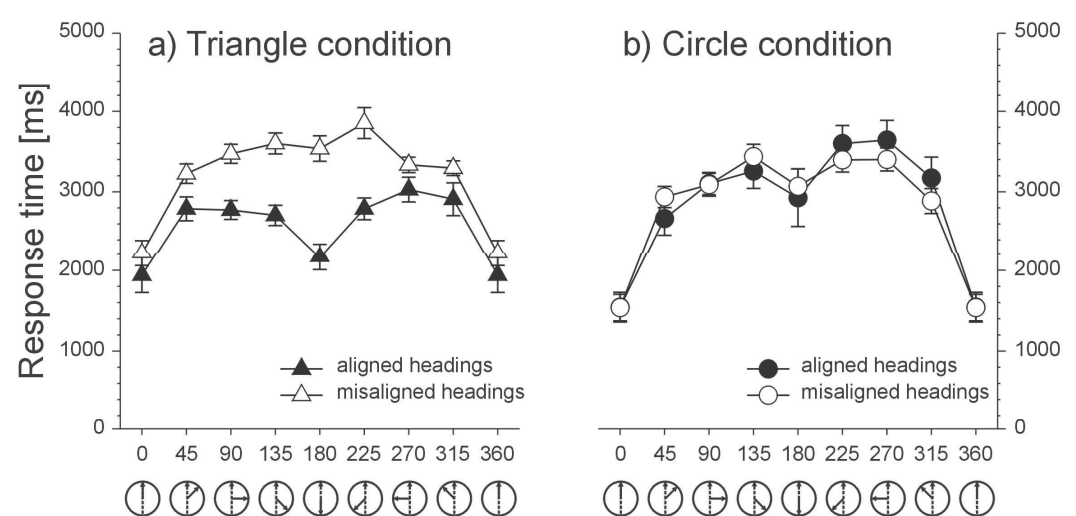

Angular deviation between target and imagined heading $\left[^{\circ}\right]$

Figure 2. Response times in Experiment 1 for retrieving object locations depending on their angular deviation from the imagined heading and on whether the heading was aligned or misaligned with allocentric reference systems. Results are shown separately for the triangle $(a)$ and the circle $(b)$ conditions. In the insets, dotted lines depict the normalized imagined heading, and arrows the retrieval direction relative to this heading. Error bars denote standard errors of the mean corrected for pure intersubject variance. The $0^{\circ}\left(=360^{\circ}\right)$ condition appears twice to enhance evaluation of symmetry.

was larger for aligned than for misaligned headings. Overall, performance was best when the imagined heading was towards the base of the triangle, followed by heading towards the top; the remaining headings did not differ systematically. There was no evidence for an alignment effect in the circle condition, $F(1,15)=0.11, p>.9$, nor for an interaction with egocentric retrieval direction, $F(7,105)=0.81, p>.5$.

In a second analysis of allocentric effects, we studied the retrieval times for objects located at different positions with respect to the triangular reference frame, this time pooling across different imagined headings. We compared objects positioned on four possible axes, each comprising two locations (Figure 3 ). We defined a symmetry axis as running through the top and the base of the triangular carpet, a perpendicular axis forming a right angle with the symmetry axis and running through the center of the surrounding circular room, and two oblique axes forming the diagonals. One-way ANOVA confirmed that axes differed in the triangle condition, $F(3,45)=17.75, p<.001$, with planned 


\section{Experiment 1}

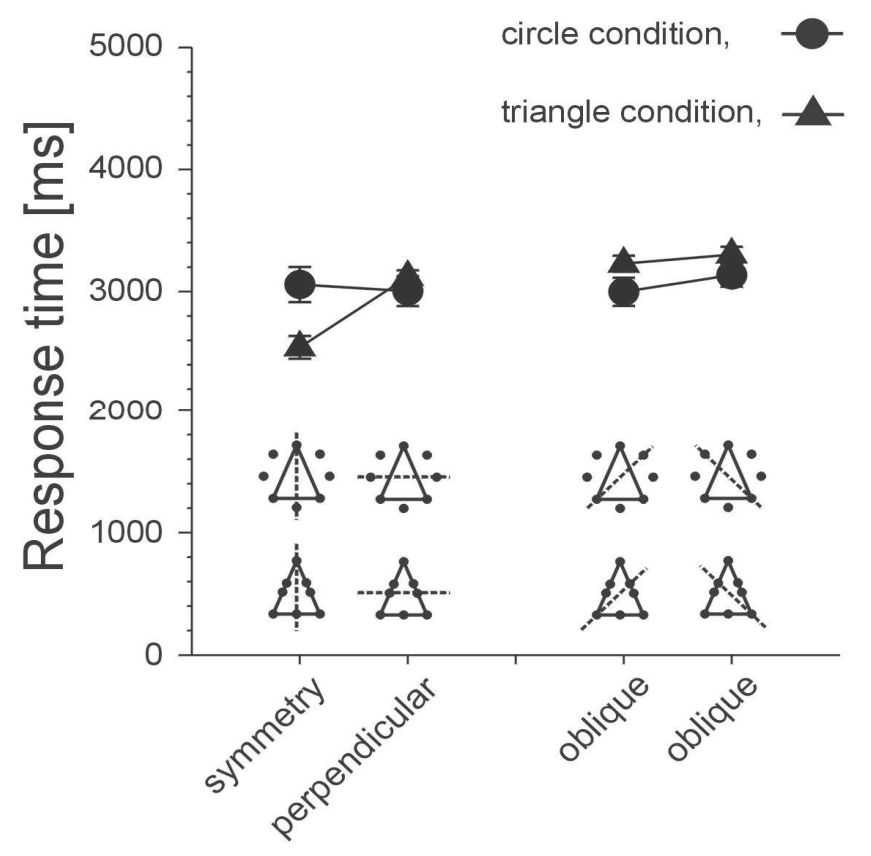

\section{Target position on environmental axis}

Figure 3. Response times in Experiment 1 depending on whether the retrieved object was on the symmetry, perpendicular, or oblique axis of the allocentric reference system, as symbolized by the insets. Error bars denote standard errors of the mean corrected for pure intersubject variance.

comparisons ( $F$ tests at $\alpha=.01$, pooled error terms) showing that objects on the symmetry axis were retrieved faster than objects on the perpendicular axis, $F(1,45)=24.81, p<.001$. In contrast, the two oblique axes did not differ from each other, $F(1,45)=0.41, p>.5$, and there was also no clear difference between the perpendicular axis and the oblique axes, $F(1,45)=2.10, p<.1$. There were no differences whatsoever among axes in the circle condition, $F(3,45)=0.21, p>.8$.

Pointing error. Analysis of signed angular pointing error confirmed the crucial role of the symmetry axis in the triangle condition. Figures 4 and 5 show the average pointing directions within their $95 \%$ confidence limits. Pointing in the circle condition (Figure 4) was essentially veridical, and the signed angular 


\section{Experiment 1}

\section{Circle condition}

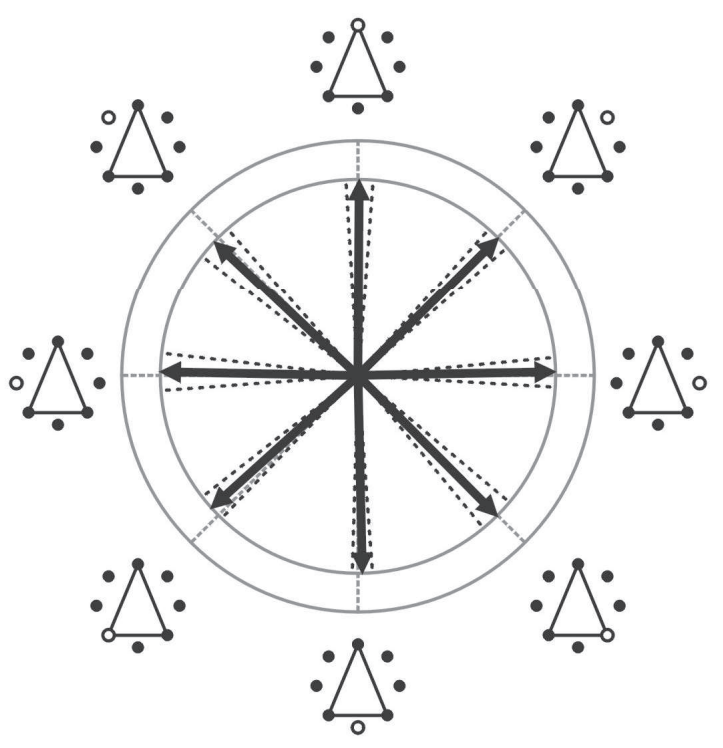

Figure 4. Pointing errors in the circle condition of Experiment 1. The polar coordinate systems shows the actual angular object positions as symbolized by the insets. Black arrows show the average pointing direction when these object positions were retrieved. Black lines flanking the arrows indicate $95 \%$ confidence limits of pointing directions.

errors (calculated by subtracting reproduced and true target positions) were homogenous, $F(7,105)=0.529, p>.60$. In contrast, some angles in the triangle condition (Figure 5) were misrepresented: Pointing directions for $45^{\circ}$ and $315^{\circ}$ targets were strongly distorted away from the symmetry axis, whereas pointing directions for $135^{\circ}$ and $225^{\circ}$ targets were slightly distorted towards it, so that the signed angular errors were strongly inhomogenous across the eight directions, $F(7,105)=20.61, p<.001$. Note that this pattern of distortions is symmetrical about the principal axis of the triangle, indicating that this line was used as a spatial reference. 


\section{Experiment 1}

\section{Triangle condition}

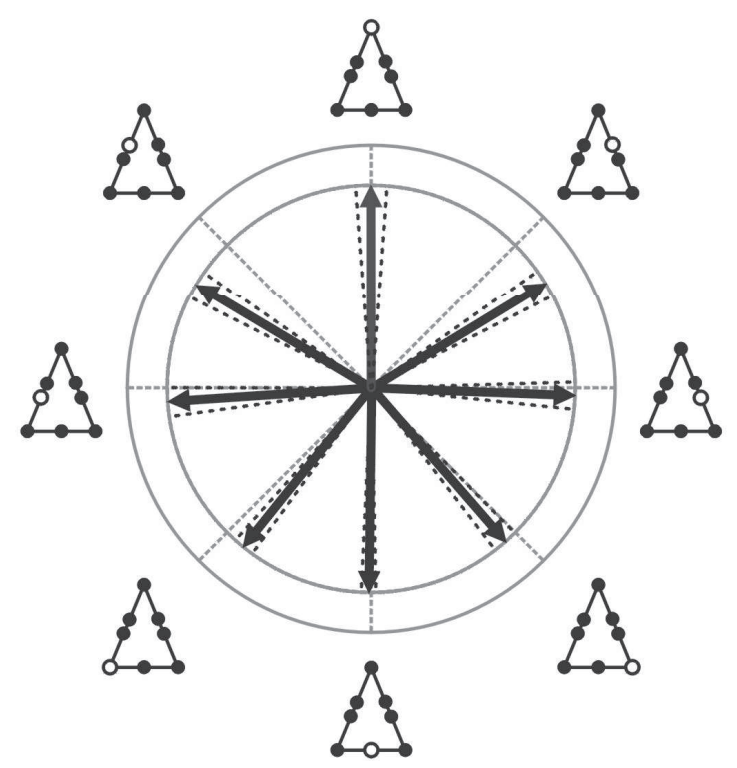

Figure 5. Pointing errors in the triangle condition of Experiment 1. Conventions as in Figure 4.

\section{Discussion}

The present data provide further evidence for an influence of egocentric as well as allocentric reference systems on spatial memory. Egocentric coding is demonstrated by the fact that responses were fastest and most accurate for objects imagined straight ahead, with response times increasing for targets located farther away from the imagined heading, and with a slight advantage for positions imagined directly behind (Hintzman et al., 1981; Werner et al., 1998). Allocentric effects are superimposed on this data pattern. Participants in the triangle condition could point to objects faster when mentally aligned with the symmetry axis of the triangular carpet, compared to other mental orientations. They were also faster in retrieving objects located on the symmetry axis of the triangle. However, the carpet itself was obviously not sufficient to establish an environmental reference frame, because participants in the circle condition were 
completely uninfluenced by it. Thus, the data support an influence of an objectcentered but not an environmental allocentric reference system.

This result is rather surprising because we had expected some influence of the carpet even in the circle condition: In the absence of any other directional cues, the carpet should have been spontaneously used to provide spatial orientation (Mou \& McNamara, 2002). Instead, memory organization seems to depend exclusively on the layout of the objects here. These data clearly show that geometric characteristics of the environment are not inevitably used in organizing spatial memory; instead, even quite conspicuous environmental reference frames may fail to enter the memory representation.

In the triangle condition, angular positions of objects were misrepresented away from the top of the triangle, whereas no such distortion was evident in the circle condition. There is evidence that memory for spatial positions is systematically distorted away from attended objects (Suzuki \& Cavanagh, 1997) or salient reference points and axes (Schmidt, 2004; Schmidt et al., 2003; Werner \& Diedrichsen, 2002), so that distortions away from the top of the triangle may indicate that this point served as a spatial landmark. Importantly, the pattern of distortions is symmetrical about the principal axis of the triangle, suggesting that this line was used as a spatial reference.

\section{Experiment 2}

Experiment 1 had failed to provide evidence for an impact of an environmental reference system. In Experiment 2, we had participants learn the locations of circularly arranged objects within an isosceles triangular room, expecting that the reference system imposed by room geometry would now be salient enough to lend an advantage to the symmetry axis of the room.

\section{Methods}

Participants. 16 right-handed students from the University of Göttingen (7 men, 9 women, age 19 to 33 years) participated for course credit or for a payment of $€ 7$. All of them gave informed consent.

Apparatus and Stimuli. The study phase took place in an isosceles triangular chamber with the same aspect ration as the triangular carpet in Experiment 1. The chamber had edge lengths of 264 and $345 \mathrm{~cm}$ and a height of $200 \mathrm{~cm}$. It was built out of thick drapery that covered the walls, floor, and ceiling and was set up in a darkened larger room. To make room geometry more conspicuous, red paper stripes were attached along the inner nooks of the chamber. The chamber was accessed through a flap in the wall that was concealed with fabric once the participant had entered. Three $60-\mathrm{W}$ overhead projectors illuminated the corners from outside to remove any effects of residual daylight that might have provided a cue as to the orientation of the triangular chamber within the 
larger room. The objects were identical to those in Experiment 1. They were arranged in a circular configuration at a radius of $156 \mathrm{~cm}$, with spacings of $45^{\circ}$ (Figure 1b).

Proscedure. The study phase was similar to that of Experiment 1. Participants were led blindfolded into the surrounding room and turned themselves until they felt disoriented; they were then led into the triangular room, were seated in the center of the circular object configuration, and the blindfold was removed. Participants then studied the locations of the eight objects, rotating themselves upon instruction from one heading to an adjacent one until the configuration had been studied eight times, once from each possible orientation, for one minute each. After that, they were allowed to study the object configuration freely. Participants were then blindfolded once more and led out of the room. Each object served as a first learning position for two participants. Each participant received a novel configuration of objects and a new orientation of the triangular room within the surrounding room. The study phase lasted about $10 \mathrm{~min}$.

The test phase was identical to Experiment 1 except that participants were verbally informed when making pointing errors of more than $\pm 90^{\circ}$. After the test phase, participants were debriefed and asked to make a drawing of the object arrangement.

\section{Results}

Four participants had to be replaced because their average response times had exceeded a predetermined criterion (mean plus one standard deviation of all participants, $4941 \mathrm{~ms})$. As before, trials with an angular deviation exceeding $\pm 90^{\circ}$ were excluded $(1.3 \%)$, as were trials with response times more extreme than \pm 3 standard deviations from the mean $(1.5 \%)$, calculated separately for each participant. ANOVAs were carried out at $\alpha=.05$.

Response times. Results were similar to the triangle condition of Experiment 1 (Figure 6). Again, effects of an egocentric reference system were analyzed by looking at the retrieval times of objects at various positions with respect to the imagined heading. There was a strong main effect of retrieval direction, $F(7$, $105)=19.05, p<.001$, indicating that retrieval was fastest for objects imagined straight ahead but slower for objects located increasingly far apart from the imagined heading (Figure 4a). Objects imagined straight ahead were retrieved faster than objects imagined from any other position (planned contrast; $F(1,15)$ $=51.57, p<.001)$. Also, objects imagined straight behind were retrieved faster than objects at neighboring $\left(135^{\circ}\right.$ and $\left.225^{\circ}\right)$ positions, $F(1,15)=5.75, p<.05$.

Again, two types of allocentric effects on response times were analyzed. First, we compared imagined headings aligned or misaligned with the triangular reference system. As predicted, retrieval was faster for aligned than for misaligned headings, $F(1,15)=19.66, p<.001$, this effect interacting only 
360 SCHMIDT, LEE

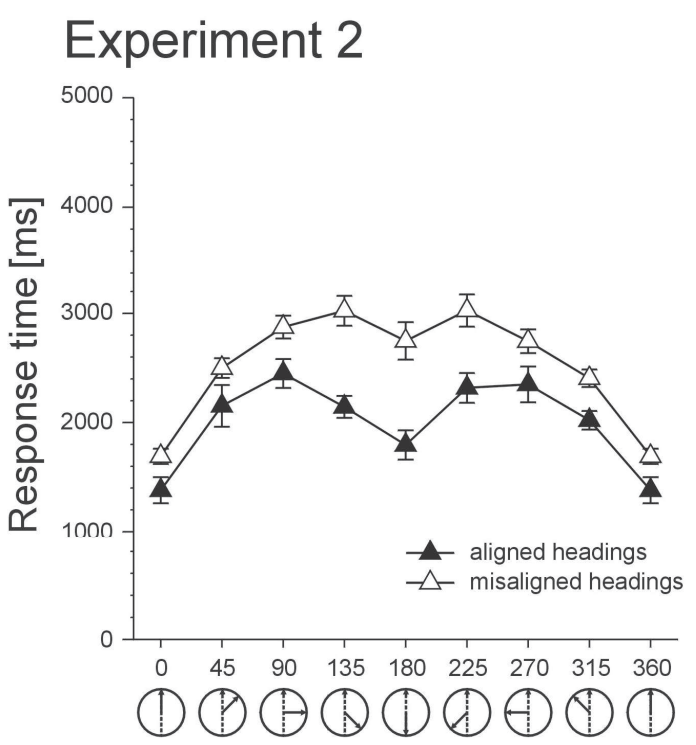

Deviation from imagined heading $\left[^{\circ}\right]$

Figure 6. Response times in Experiment 2 for retrieving object locations depending on their angular deviation from the imagined heading and on whether the heading was aligned or misaligned with the environmental reference system. Error bars denote standard errors of the mean corrected for pure intersubject variance. The $0^{\circ}\left(=360^{\circ}\right)$ condition appears twice to enhance evaluation of symmetry.

marginally with egocentric retrieval direction, $F(7,105)=2.41, p=.058$. Overall, performance was best when the imagined heading was towards the base or top of the triangle; the remaining headings did not differ systematically. Second, we compared retrieval times for objects located on the symmetry, perpendicular, and oblique axes with respect to the triangular reference system (Figure 7), pooling across imagined headings. One-way ANOVA confirmed significant differences between axes, $F(3,45)=10.63, p<.001$. Planned comparisons (at $\alpha / 3=.017$ ) confirmed that objects on the symmetry axis were retrieved faster than objects from the perpendicular axis, $F(1,45)=6.73$, $p=.013$, and that retrieval from the perpendicular axis was faster than from the oblique axes, $F(1,45)=6.74, p=.013$. The two oblique axes did not differ from each other, $F(1,45)=0.01, p>.9$. 


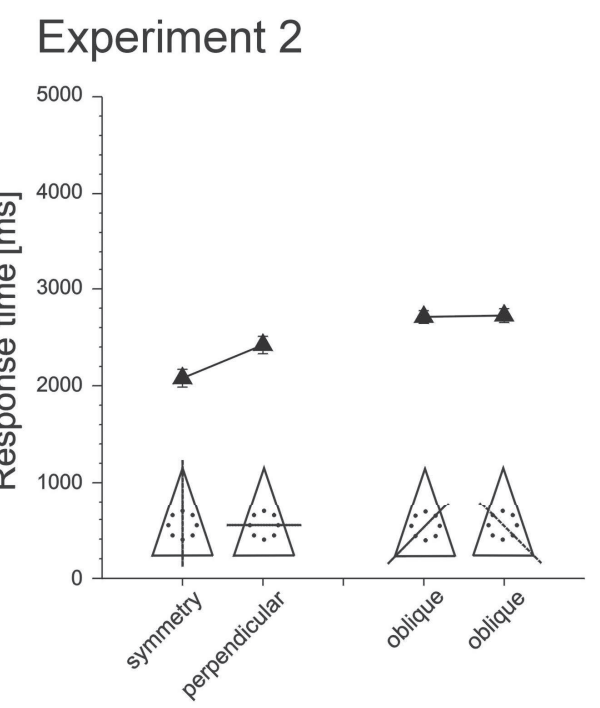

Target position on environmental axis

Figure 7. Response times in Experiment 2 depending on whether the retrieved object was on the symmetry, perpendicular, or oblique axis of the allocentric reference system, as symbolized by the insets. Error bars denote standard errors of the mean corrected for pure intersubject variance.

Pointing error. Analysis of signed pointing error revealed some spatial misrepresentation for pointing towards $45^{\circ}$ and $315^{\circ}$ targets, which were slightly distorted away from the symmetry axis (Figure 8). This finding would corroborate our conclusions from the retrieval time data that the allocentric symmetry axis had a special role in organizing spatial memory. However, the effect was much smaller than in the triangle condition of Experiment 1, and did not quite reach significance, $F(7,105)=2.49, p=.073$.

\section{Discussion}

Participants were faster when retrieving objects close to their imagined heading or objects imagined directly behind them, thus replicating the results from Experiment 1 and earlier studies (e.g., Hintzman et al., 1981; Werner et al., 1998). In contrast to Experiment 1, the environmental reference system imposed by room geometry sufficed to influence the spatial memory representation even with a neutrally arranged object configuration: Participants pointed to objects substantially faster when mentally aligned with the symmetry axis of the 


\section{Experiment 2}

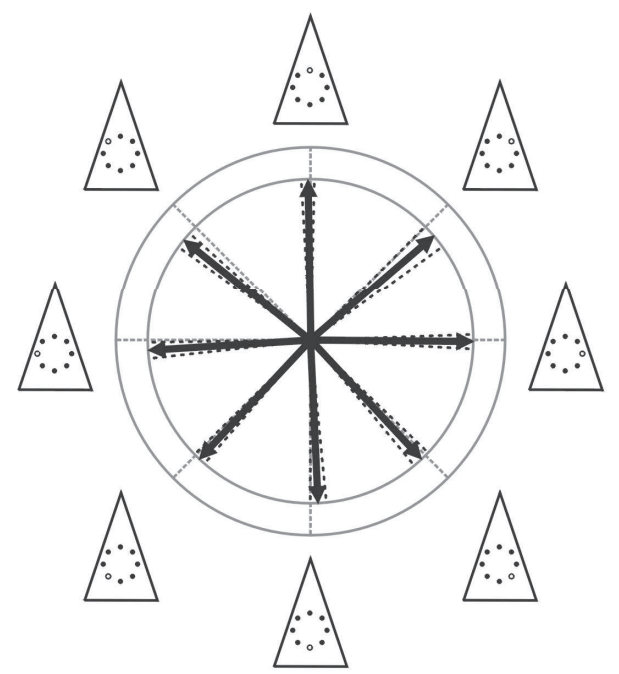

Figure 8. Pointing errors in Experiment 2. Conventions as in Figure 4.

triangular room (either towards the top or the base) than when misaligned, this allocentric effect being superimposed on the egocentric one. In addition, participants were faster when retrieving objects located on the symmetry axis of the triangular room, irrespective of imagined heading.

Although object positions adjacent to the top of the triangle were again misremembered away from it, this effect was much smaller than in the triangle condition of Experiment 1. This indicates that the triangular arrangement of objects along the edges of the carpet was crucial for spatial distortions to occur, which would suggest that the distortion in Experiment 1's triangle condition was driven by the object-centered rather than the environmental reference system. However, a slight misrepresentation of the $45^{\circ}$ and $315^{\circ}$ positions is still discernible, supporting the idea that the top of the triangle might have served as a repulsory spatial landmark in an environmental reference system (Schmidt et al., 2003).

\section{General Discussion}

All experimental conditions reported in this paper confirm strong effects of egocentric reference systems: The speed of memory retrieval critically depends on the angular distance of the target from the imagined orientation, with a 
special advantage for objects imagined directly in front of or behind the own body (Hintzman et al., 1981; Werner et al., 1998). Allocentric effects are superimposed on these egocentric ones. In a circular room (Experiment 1), the introduction of a triangular carpet failed to induce an environmental reference system; however, when the objects were arranged along the edges of the carpet so that the object-centered reference system of the target array coincided with the environmental reference system, clear advantages in response times resulted when participants imagined themselves mentally aligned with the top or the base of the triangle. In addition, objects located on the symmetry axis of the triangle were retrieved faster, irrespective of mental orientation. Similar effects were observed for circular object arrangements in a triangular room (Experiment 2), showing that a sufficiently salient environmental reference system is able to organize spatial memory even in the absence of a strong object-centered reference system. ${ }^{2}$ In sum, both experiments provide clear evidence for the simultaneous use of egocentric, object-centered, and environmental reference systems in spatial memory, supporting the idea that spatial structures are encoded by using allocentric reference cues provided by the object configurations as well as geometrical features of the learning environment, and can be recalled in terms of an egocentric reference system defined by the imagined position and orientation of the observer within the memory representation (Werner et al., 1998). Our results are consistent with previous investigations of large-scale environments showing that spatial memory is influenced by geometrical features of a park (McNamara, Rump \& Werner, 2003), a city (Werner \& K. Schmidt, 1999), a university campus (Sholl, 1987), the direction of gravity (Hubbard, 1995; Finkelmeyer, 2001), or the direction of slant in a forest environment (Werner \& Schmidt, 2006).

A novel finding here is that allocentric reference systems are able to induce systematic distortions of the remembered object positions: Angular object positions were misrepresented away from the top of the triangle, especially those neighboring the top; and only positions on the triangle's symmetry axis were remembered veridically. Symmetry of distortional fields about the principal axis of the triangle supports the notion that this axis was used as a spatial reference (Schmidt, 2004). The observed distortions also highlight a potential methodological problem with paradigms where participants have to reproduce the positions of objects from an imagined viewpoint outside the object array (e.g., Mou \& McNamara, 2002; Shelton \& McNamara, 2001, 2004). If any configuration of objects is systematically distorted in spatial

${ }^{2}$ Mou and McNamara (2002) suggest that the task-relevance of allocentric reference systems might be crucial for allocentric effects to occur. Our findings show that allocentric effects can occur spontaneously even if the allocentric structure of the object configuration or the environment is task-irrelevant. 


\section{SCHMIDT, LEE}

memory, this leads to systematic pointing biases, i.e., a systematic shift in the average pointing vector to each individual object (constant error). In contrast, uncertainty about the correct pointing direction would lead to increased variance in pointing directions (variable error). In paradigms where the object configuration is remembered from outside the object array, both types of error strongly depend on the observer's (imagined) position: For example, remembering one's own position as too close to the array would coerce use of a wider range of pointing angles, and remembering it laterally shifted would introduce effects of parallax by changing pointing angles differently for near and far objects. It is therefore advantageous to use methods that are able to detect systematic distortions in the remembered positions of the self and target objects. This is geometrically possible whenever all targets are tested from at least two sufficiently dissimilar imagined viewpoints, and our paradigm is especially adapted for this purpose.

The distortion problem is often overlooked in spatial memory research because many studies do not distinguish between constant and variable error but combine them into a single performance measure, unsigned pointing error. Random fluctuations in pointing directions lead to positive bias of this measure because they can only increase but not decrease its expected value -- in fact, the expectancies of unsigned and signed pointing error are equal only if pointing variance is zero. However, it is theoretically interesting whether pointing errors arise from spatial distortions in memory representations or from differences in noise and uncertainty, and systematic distortions might reveal interesting properties (e.g., symmetries) of the underlying reference systems. Therefore, we strongly advocate the use of signed instead of unsigned pointing error.

Our results rule out an alternate explanation of the egocentric effect, namely that participants scanned through a list of object positions learned in sequence. First, the finding that objects imagined straight behind the own body are retrieved faster than neighboring objects runs counter to this explanation, which would predict this position to be retrieved slowest. (Note that it also contradicts an explanation in terms of "mental rotation"; (Georgopoulos, Lurito, Petrides, Schwartz, \& Massey, 1989; Shepard \& Metzler, 1971). Second, list scanning should neither be affected by the mental orientation within the allocentric reference system, nor by the position of the objects with respect to that reference system.

The observation of allocentric effects in Experiment 2 also rules out a possible confound in Experiment 1. In that experiment, preserving the angular distances between objects in the triangle condition led to unequal spacings of objects along the edges of the triangular carpet, such that the $45^{\circ}$ and $315^{\circ}$ positions were closer in Euclidean distance to the $90^{\circ}$ or $270^{\circ}$ positions than to the $0^{\circ}$ position (Figure 1a). These unequal spacings might be the cause of the observed distortion of pointing movements, because interobject distances might 
have been misrepresented in spatial memory (Hollands \& Dyre, 2000). Indeed, distortions mainly arose when an object-centered reference system was present where the target objects themselves were arranged in a triangular pattern; distortions were slight (Experiment 2) or absent (Experiment 1) when the actual configuration of objects was circular. In other words, distortions occurred only when the object spacing was irregular, maybe because participants noticed that the regular arrangements were spaced in $45^{\circ}$ steps so that they could safeguard against pointing errors. Regardless, unequal spacings could not explain the egocentric effect: This effect is calculated by pooling data from all imagined headings, so that spacing effects are not confounded with egocentric pointing direction. For the same reason, unequal object spacing cannot be responsible for the allocentric effect of object position (i.e., whether objects were placed on a symmetry, perpendicular, or oblique axis), nor for the effect of imagined heading being aligned or misaligned with the triangular reference system. Indeed, the only ambiguity in Experiment 1 is whether the presence of allocentric effects in only the triangle condition is due to the triangular arrangement of objects or to their unequal spacing. However, this is nicely resolved by Experiment 2 where strong allocentric effects were found with evenly spaced objects.

Our results are in line with Mou and McNamara's (2002) suggestion that participants may spontaneously segment an object configuration in terms of any reference system that seems salient or convenient to them. If this is true, the versatility of strategic behavior makes it doubtful that a model of spatial reference systems can be developed working from the layout of the physical stimuli alone. Instead, the systematic manipulation or exclusion of individual encoding strategies would be crucial for understanding how multiple spatial reference systems are dynamically used, disused, or combined.

\section{Acknowledgments}

We thank Steffen Werner who gave the original incitation for these experiments, and Björn Rump who provided technical assistance with the soundfiles and joysticks. This work was supported by a grant from the German Science Foundation (We 1973/3) to Steffen Werner. Correspondence may be sent to either author (thomas.schmidt@psychol.uni-giessen.de, eyl7r9@mizzou.edu).

\section{References}

Burgess, N., Spiers, H. J., \& Paleologou, E. (2004). Orientational manoeuvres in the dark: Dissociating allocentric and egocentric influences on spatial memory. Cognition, 94, 149-166. 
Carlson-Radvansky, L. A., \& Irwin, D. A. (1993). Frames of reference in vision and language: Where is above? Cognition, 46, 223-244.

Connor, C. E., Preddie, D. C., Gallant, J. L., \& Van Essen, D. C. (1997). Spatial attention effects in macaque area V4. Journal of Neuroscience, 17, 32013214.

Diedrichsen, J., \& Werner, S., Schmidt, \& Trommershäuser, J. (2004). Immediate spatial distortion of pointing movements induced by visual landmarks. Perception \& Psychophysics, 66, 89-103.

Diwadkar, V. A., \& McNamara, T. P. (1997). Viewpoint dependence in scene recognition. Psychological Science, 8, 302-307.

Finkelmeyer, A. (2001). Die rolle der gravitation beim aufbau und abruf räumlichen wissens. Unpublished master's thesis, Unviversity of Göttingen.

Flanders, M., Helms Tillery, S. I., \& Soechting, J. F. (1992). Early stages in a sensorimotor transformation. Behavioral \& Brain Sciences, 15, 309-362.

Franklin, N., \& Tversky, B. (1990). Searching imagined environments. Journal of Experimental Psychology: General, 119, 63-76.

Franklin, N., Tversky, B., \& Coon, V. (1992). Shifting points of view in spatial mental models. Memory \& Cognition, 20, 507-518.

Georgopoulos, A. P., Lurito, J. T., Petrides, M. Schwartz, A. B., \& Massey, J. T. (1989). Mental rotation of the neuronal population vector. Science, 243, 234 236.

Hermer, L., \& Spelke, E. (1994). A geometric process for spatial reorientation in young children. Nature, 370, 57-59.

Hermer, L., \& Spelke, E. (1996). Modularity and development: The case of spatial reorientation. Cognition, 61, 195-232.

Hintzman, D. L., O'Dell, C. S., \& Arndt, D. R. (1981). Orientation in cognitive maps. Cognitive Psychology, 13, 149-206.

Hollands, J. G., \& Dyre, B. P. (2000). Bias in proportion judgments: The cyclical power model. Psychological Review, 107, 500-524.

Hubbard, T. L. (1995). Cognitive representation of motion: Evidence for friction and gravity analogues. Journal of Experimental Psychology: Learning, Memory, \& Cognition, 21, 241-254.

Huttenlocher, J., Hedges, L. V., \& Duncan, S. (1991). Categories and particulars: Prototype effects in estimating spatial location. Psychological Review, 98, 352-376.

Jainek, V. (2001). Der Einfluß natürlicher Referenzsysteme auf die Verfügbarkeit räumlichen Wissens am Beispiel des Gefälles eines Hanges. Unpublished master's thesis, University of Göttingen.

Laeng, B., Peters, M., \& McCabe, B. (1998). Memory for locations within regions: Spatial biases and visual hemifield differences. Memory \& Cognition, 26, 97-107. 
Levinson, S. (1996). Frames of reference and Molyneux's question: Crosslinguistic evidence. In P. Bloom, M. A. Peterson, L. Nadel, \& M. Garrett (Eds.), Language and space (pp. 109-169). Cambridge, MA: MIT Press.

Levine, M., Jankovic, I. N., \& Palij, M. (1982). Principles of spatial problem solving. Journal of Experimental Psychology: General, 111, 157-175.

McNamara, T. P., Rump, B., \& Werner, S. (2003). Egocentric and geometric frames of reference in memory of large-scale space. Psychonomic Bulletin \& Review, 10, 589-595.

Moran, J., \& Desimone, R. (1985). Selective attention gates visual processing in the extastriate cortex. Science, 229, 782-784.

Mou, W., \& McNamara, T. P. (2002). Intrinsic frames of reference in spatial memory. Journal of Experimental Psychology: Learning, Memory, \& Cognition, 28, 162-170.

Mou, W., McNamara, T. P., Valiquette, C. M., \& Rump, B. (2004). Allocentric and egocentric updating of spatial memories. Journal of Experimental Psychology: Learning, Memory, \& Cognition, 30, 142-157.

Mou, W., Zhang, K., \& McNamara, T. P. (2004). Frames of reference in spatial memories acquired from language. Journal of Experimental Psychology: Learning, Memory, \& Cognition, 30, 171-180.

Milner, A. D., \& Goodale, M. A. (1995). The Visual Brain in Action. Oxford: Oxford University Press.

Nelson, T. O., \& Chaiklin, S. (1980). Immediate memory for spatial location. Journal of Experimental Psychology: Human Learning and Memory, 6, 529545.

Pani, J. R., \& Dupree, D. (1994). Spatial reference systems in the comprehension of rotational motion. Perception, 23, 929-946.

Roskos-Ewoldsen, B., McNamara, T. P., Shelton, A. L., \& Carr, W. (1998). Mental representations of large and small spatial layouts are orientation dependent. Journal of Experimental Psychology: Learning, Memory, \& Cognition, 24, 215-226.

Schmidt, T. (2004). Spatial distortions in visual short-term memory: Interplay of intrinsic and extrinsic reference systems. Spatial Cognition \& Computation, 4, 313-336.

Schmidt, T., Werner, S., \& Diedrichsen, J. (2003). Spatial distortions induced by multiple visual landmarks: How local distortions combine to produce complex distortion patterns. Perception \& Psychophysics, 65, 861-873.

Shelton, A. L., \& McNamara (1997). Multiple views of spatial memory. Psychonomic Bulletin \& Review, 4, 102-106.

Shelton, A. L., \& McNamara, T. P. (2001). Systems of spatial reference in human memory. Cognitive Psychology, 43, 274-310. 
Shelton, A. L., \& McNamara, T. P. (2004). Orientation and perspective dependence in route and survey learning. Journal of Experimental Psychology: Learning, Memory, \& Cognition, 30, 158-170.

Shepard, R. N., \& Metzler, J. (1971). Mental rotation of three-dimensional objects. Science, 171, 701-703.

Sholl, M. J. (1987). Cognitive maps as orienting schemata. Journal of Experimental Psychology: Learning, Memory, \& Cognition, 13, 615-628.

Spitzer, H., Desimone, R., \& Moran, J. (1988). Increased attention enhances both behavioral and neural performance. Science, 240, 338-340.

Suzuki, S., \& Cavanagh, P. (1997). Focused attention distorts visual space: An attentional repulsion effect. Journal of Experimental Psychology: Human Perception \& Performance, 23, 443-463.

Tolman, E. C. (1948). Cognitive maps in rats and men. Psychological Review, 55, 189-208.

Trommershäuser, J., \& Schmidt, T. (2003). Attention controls spatial distortions in visual short-term memory. Manuscript in preparation.

Wang, R. F., \& Brockmole, J. R. (2003). Simultaneous spatial updating in nested environments. Psychonomic Bulletin \& Review, 10, 981-986.

Wang, R. F., \& Simons, D. J. (1999). Acitive and passive scene recognition across views. Cognition, 70, 191-210.

Werner, S., \& Diedrichsen, J. (2002). The time course of spatial memory distortions. Memory \& Cognition, 30, 718-730.

Werner, S., Saade, C., \& Lüer, G. (1998). Relations between the mental representation of extrapersonal space and spatial behavior. In K. F. Wender, C. Freksa, \& C. Habel (Eds.), Spatial cognition (pp. 108-127). Berlin: Springer.

Werner, S., \& Schmidt, K. (1999). Environmental reference systems for largescale spaces. Spatial Cognition \& Computation, 1, 447-473.

Werner, S., \& Schmidt, T. (2006). Impact of natural reference systems on spatial memory organization. Manuscript in preparation. 